\title{
SCIENTIFIC REPORTS

\section{OPEN 3D-printable portable open-source platform for low-cost lens-less holographic cellular imaging}

\author{
Stephan Amann (D), Max von Witzleben \& Stefan Breuer
}

Received: 1 March 2019
Accepted: 22 July 2019

Published online: 02 August 2019

\begin{abstract}
Digital holographic microscopy is an emerging, potentially low-cost alternative to conventional light microscopy for micro-object imaging on earth, underwater and in space. Immediate access to micronscale objects however requires a well-balanced system design and sophisticated reconstruction algorithms, that are commercially available, however not accessible cost-efficiently. Here, we present an open-source implementation of a lens-less digital inline holographic microscope platform, based on off-the-shelf optical, electronic and mechanical components, costing less than \$190. It employs a BluRay semiconductor-laser-pickup or a light-emitting-diode, a pinhole, a 3D-printed housing consisting of 3 parts and a single-board portable computer and camera with an open-source implementation of the Fresnel-Kirchhoff routine. We demonstrate $1.55 \mu \mathrm{m}$ spatial resolution by laser-pickup and $3.91 \mu \mathrm{m}$ by the light-emitting-diode source. The housing and mechanical components are 3D printed. Both printer and reconstruction software source codes are open. The light-weight microscope allows to image label-free micro-spheres of $6.5 \mu \mathrm{m}$ diameter, human red-blood-cells of about $8 \mu \mathrm{m}$ diameter as well as fast-growing plant Nicotiana-tabacum-BY-2 suspension cells with $50 \mu \mathrm{m}$ sizes. The imaging capability is validated by imaging-contrast quantification involving a standardized test target. The presented 3D-printable portable open-source platform represents a fully-open design, low-cost modular and versatile imaging-solution for use in high- and low-resource areas of the world.
\end{abstract}

Digital inline holography (DIHM) is an imaging modality within the fast evolving field of imaging microscopy research since a few decades ${ }^{1}$ and is based on Gabor's holographic principle ${ }^{2}$. By wide-field illumination of semi-transparent nano- to micron-sized objects with either a coherent or incoherent point light source, an interference pattern forming the hologram is created within a detection plane. It consists of the scattered part of the beam, object beam, and the unscattered (transmitted) part, the reference beam. The hologram contains amplitude and phase information of the imaged objects and allows for a numerical reconstruction of the object's light field. To date, digital holography has been pioneered across a broad range of the electromagnetic spectrum: Terahert $z^{3}$ and infrared ${ }^{4}$ holography bear the attractive potential to penetrate opaque media, whereas $\mathrm{UV}^{5,6}$ and $\mathrm{X}-\mathrm{ray}^{7,8}$ illumination enable imaging on nanometer scale. Electron holography today is a mature research field and widely in use, allowing for molecular imaging ${ }^{9,10}$. Digital holography within the visible wavelength regime has attracted considerable attention where a broad spectrum of light sources has been employed, ranging from gas $^{11,12}$, solid state ${ }^{13}$ and semiconductor ${ }^{1,14-16}$ lasers over light emitting diodes (LEDs) ${ }^{17-20}$ to halogen lamps ${ }^{21}$. Recently, ultra-broadband digital holography with sunlight illumination has been demonstrated, employing a new reconstruction algorithm ${ }^{22}$. In digital off-axis holography $(\mathrm{DOAH})$, reference and object beam are spatially separated and recombine at an angle within the detector plane. Such experiment can already be compact, combining for example a collimation lens and two gradient-index lenses forming the point-light source and which enables a highly stable off-axis digital holographic system ${ }^{23}$. This helps reducing twin images, typically a challenge in holography set-ups ${ }^{24}$. During reconstruction, a conjugate out of focus image of the object is obtained in addition to the reconstructed object. This can lead to residual fringes, decreased contrast and an over all reduced image quality. In DOAH, object and twin image can be separated in the Fourier domain of the hologram. DOAH set-ups require a rather large number of optical components including mirrors, beam splitters and collimation lenses. Complementary to DOAH, DIHM constitutes a compact holography implementation that avoids to spatially separate the reference beam from the object beam. DIHM setups typically comprise of a coherent or an incoherent

Institute for Applied Physics, Technische Universität Darmstadt, Schlossgartenstraße 7, 64289, Darmstadt, Germany. Correspondence and requests for materials should be addressed to S.B. (email: stefan.breuer@physik. tu-darmstadt.de) 
point light source, a 2D digital detector array and, positioned in between, the micron-scale object sample to be imaged. It has been demonstrated in the ultraviolet ${ }^{5}$, visible ${ }^{13}$, near-infrared ${ }^{25}$ up to the mid-infrared ${ }^{26}$ wavelength regime. In DIHM, only a negligible influence of the twin image on the quality of microscopic object analysis has been demonstrated ${ }^{1,27}$, as the twin image is defocused across the whole detector array when the distance between detector and object is large as compared to the object size. Moreover, for DIHM several approaches to numerically remove the twin image from the reconstructed image have been demonstrated ${ }^{28-30}$. Ideal light sources are semiconductor photonic emitters such as laser diodes (LDs) and LEDs thanks to their compactness, high electro-optical efficiencies, comparable low price and availability. Partially coherent light sources can even enhance the result in DIHM as coherent speckle noise is reduced ${ }^{27}$ while being less susceptible to mechanical vibrations. Considering the generally less complex implementation, easy replacement, lower price, high reliability and reduced safety issues, LEDs appear as ideal DIHM light sources for student and early researcher education. To ensure homogeneous illumination, the LED or LD light can be coupled into an optical fibre ensuring a Gaussian beam profile at the fibre's end ${ }^{15,19,20,31,32}$. In addition, more compact or complex experimental set-ups can be realized thanks to the mechanical fibre flexibility ${ }^{31}$. Both charge-coupled device (CCD) and complementary metal-oxide-semiconductor (CMOS) cameras are commonly used as detectors, with an increasing number of CMOS chips due to recent advances in terms of sensitivity and reduced pixel size. Two parallel tracks of current DIHM research can be identified, focusing on two different detection schemes: On-chip microscopy on the one hand, where the sample under investigation is located close to the CMOS detector. Its advantage is the large field-of-view which corresponds to the whole detector area. Several on-chip microscopes employing low-coherent LED illumination ${ }^{17,31}$ have been demonstrated making them already cost-efficient and easy to operate. In combination with several pixel super-resolution approaches using multi-height imaging ${ }^{19}$, wavelength scanning ${ }^{20}$, sub-pixel shifting ${ }^{33}$ or flowing samples ${ }^{34}$, sub-micron spatial resolution is possible. Sub cellular imaging of malaria infected blood cells ${ }^{35}$, red blood cell (RBC) imaging with a cell phone camera ${ }^{18}$ and colour imaging using $\mathrm{DIHM}^{31}$ have been reported. On the other hand, fringe magnification technique are studied where the sample is located near the point source ${ }^{1,15,23}$. Here, the maximum lateral resolution $\delta_{\text {lat }}$ is accompanied by a decreased field-of-view (see equation (5)).

DIHM research has already reached the stage of commercialization where for example several DIHM implementations exist including lens-less inline and DOAH schemes ${ }^{36}$ delivering lateral resolutions of $0.3 \mu \mathrm{m}$. A submersible holographic microscope for remote in-vivo oceanic microscopy has been reported ${ }^{37}$ and is now commercially available ${ }^{38}$. Moreover, lens-based DOAH has recently been employed in in-line industrial production control ${ }^{39}$ and for imaging of semiconductor structures ${ }^{40}$. In life-sciences, DIHM has the advantage of working label-free, which enables non-invasive, in-vivo study of biological samples, for example RBCs ${ }^{41-43}$, parasited mouse $\mathrm{RBCs}^{44}$, sperm cells ${ }^{15}$, diarrhea parasites ${ }^{17}$, and several aquatic organisms ${ }^{37}$. Moreover, as information about the whole sample volume is encoded in one single hologram, processing speed is substantially increased as compared to microscopic scanning techniques such as confocal or fluorescence microscopy. Thus, large sample volumes can be analyzed efficiently, such as cell cultures of human cancer cells ${ }^{45,46}$ as well as microplastic pollution in marine environments ${ }^{47}$. Furthermore, by acquiring and analyzing sequences of images, DIHM enables long term cell evolution studies ${ }^{32}$ and micro-particle tracking ${ }^{48}$. Although differently complex, portable and low-cost DIHM solutions have recently been reported ${ }^{15,18,49,50}$, critically important details necessary for their realization in a laboratory are mostly disclosed. Such important details include details on employed light sources, distances between objects and light source or detector, methods of reconstruction, non obvious limitations or design or construction files thus making it challenging to set up a DIHM without too much prior expertise and avoids access to low-cost micron spatial resolution imaging. In this work first, we present two experimental DIHM platforms employing an LED and a LD as illumination sources and operate both by a portable single-board computer and camera. The LD has been disassembled from a standard commercially available Blu-ray disk drive. The housing and all mechanical mounts are 3D printed ${ }^{51}$. Second, we describe the implemented open-source hologram reconstruction based on $\mathrm{HoloPy}^{52}$ and Fiji plugin ${ }^{53}$. All employed code is open-source accessible aiming at triggering further developments and sharing between research laboratories, diagnostic labs and for science education. Third, we demonstrate microscopic 2D-imaging of polystyrene micro-spheres (PMSs) and mature human RBCs with micron spatial resolution. Fourths, we perform 2D-imaging of larger tobacco BY-2 cells (TBY2s). Fifths, we quantify the achieved spatial resolution by optical contrast analysis of an USAF microscopic imaging test target. Sixths, we summarize our efforts in developing a 3D-printable open-source platform for cellular imaging and provide a brief outlook on our next activities.

\section{Laboratory and 3D Printed Opto-Mechanical DIHM Setups}

Both developed DIHM implementations, described in the following, aim at achieving and validating the maximum achievable spatial resolution when an temporally and spatially coherent source as well as a temporally and spatially incoherent semiconductor light source at equivalent emission wavelengths are considered. First, a LD-based lens-less DIHM platform is developed that aims at demonstrating single-digit micrometer spatial resolution cellular imaging by a temporally coherent source and is schematically depicted in Fig. 1(a). It employs a $405 \mathrm{~nm}$ emitting LD in a laser-pickup which has been dismounted from a commercially available standard Blu-ray disc drive. The laser emission is coupled into a standard single-mode fibre. The diverging fundamental Gaussian mode beam is directed towards the object glass plate at a distance of $24.09 \mathrm{~mm}$ from the fibre exit facet. The CMOS camera is positioned at a distance of $f=30 \mathrm{~mm}$. Second, an LED-based lens-less DIHM platform, depicted schematically in Fig. 1(c), is designed and constructed by 3D printable parts, with the over all system costs amounting to less than $\$ 190$. Compared to Fig. 1(a), in Fig. 1(b) a $430 \mathrm{~nm}$ emitting high-power LED is employed as a temporally and spatially incoherent semiconductor light source. A fraction of the emitted light is passed through a high-precision pinhole where $1.1 \mu \mathrm{W}$ of optical power are emitted at the pinhole for an injection current of $125 \mathrm{~mA}$ and impinge on objects to be imaged $(z=5.91 \mathrm{~mm})$. For live cell imaging, such ultra-low 

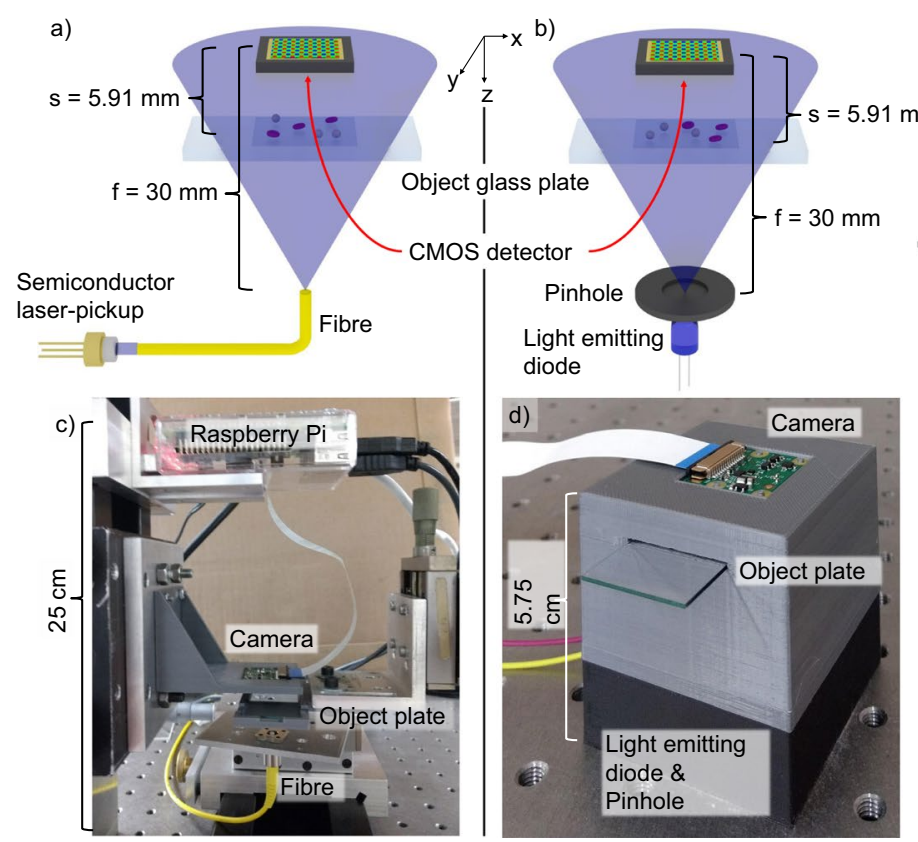

e)
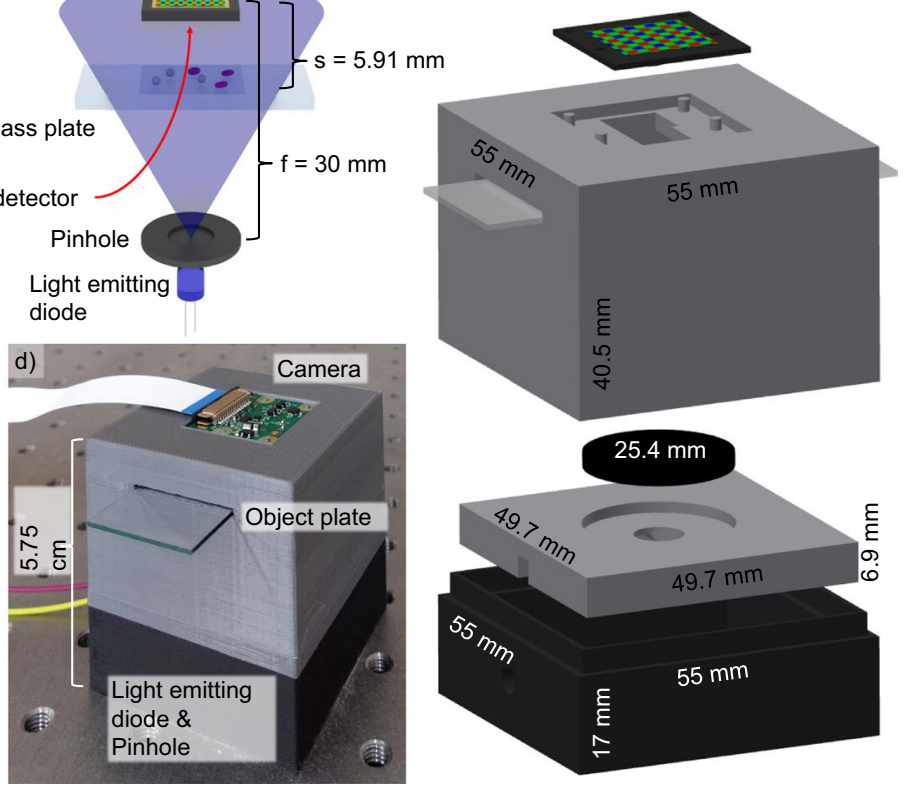

Figure 1. Developed laboratory DIHM and 3D printable DIHM setup. Schematics of the (a) fibre-coupled LD pickup inline illumination. (b) LED pinhole setup. (c) Image of the laboratory fibre-coupled laser-pickup setup and dimensions. (d) Image of the complete 3D-printed LED set-up. (e) Exploded view of the three developed and used 3D printed parts with dimensions (CAD files open accessible, see methods chapter). Schematically depicted light propagation not to scale.

optical power is of critical importance, as cell damage by light exposure needs to be minimised. To construct the LED-based platform in Fig. 1(b), first three mechanical parts in Fig. 1(c) are 3D-printed, see section "Methods", and assembled as sketched in Fig. 1(d,e) where also specific dimensions of the platform are depicted. For both experiments, equal spatial separations between emission facet, microscope glass plate, carrying the micro-objects under investigation, and CMOS detector are chosen. A distance of $30 \mathrm{~mm}$ between light source and detector is selected in order to reach a compact experimental set-up, while still maintaining illumination of the hole detector area. The position of the object emerges from resolution optimization, see section "Resolution". A Raspberry Pi single-board portable computer and a Raspberry Pi CMOS detector camera with a pixel size of $(1.12 \times 1.12) \mu \mathrm{m}^{2}$ serve as light source current injection driver and hologram acquisition, see section "Methods". A constant injection current of $28 \mathrm{~mA}$ for the LD were provided by a commercial LD driver, while a current of $125 \mathrm{~mA}$ for the LED was provided by a simple electrical circuit made of off the shelf components. A conventional rechargeable power bank battery pack provides electrical energy for the computer. Estimated theoretical spatial resolutions of $\delta_{\mathrm{LD}}=0.87 \mu \mathrm{m}$ with a LD source and $\delta_{\mathrm{LED}}=0.92 \mu \mathrm{m}$ with a LED light source at an available CMOS pixel size of $1.12 \mu \mathrm{m}$ and at a distance of $f=30 \mathrm{~mm}$ between fibre facet and CMOS detector are expected for the LD and LED light source, respectively.

\section{Image Reconstruction}

Following the hologram acquisition, information retrieval of the cellular objects deposited on the object glass plate is performed numerically. The propagation of light fields is completely described by diffraction theory. Hence it is possible to reconstruct amplitude and phase information of the objects from their interference patterns generated on the camera. At the object location the pattern is focused and reveals the shape and morphology of micro-objects. Numerically, arbitrary planes can be re-focused in retrospect yielding access to volumes with a large number of objects in different heights in z-direction which can be studied with acquiring a single image. The propagation of a wave front towards the detector is described by the Fresnel-Kirchhoff diffraction integral

$$
U_{\mathrm{det}}(X, Y)=-\frac{i}{\lambda} \iint U_{\mathrm{in}}(x, y) t(x, y) \frac{\exp (i k|\vec{r}-\vec{R}|)}{|\vec{r}-\vec{R}|} d x d y,
$$

where $U_{\text {det }}(X, Y)$ denotes the wave field on the detector, $U_{\text {in }}(x, y)$ is the incident wave, $t(x, y)$ the transmission function of the object, and $\vec{r}=(x, y, z)$ and $\vec{R}=(X, Y, 0)$ are two points in the object plane respectively detector plane ${ }^{54}$. The amplitude and phase distribution of the object can be obtained via an inverse integral

$$
U_{\mathrm{obj}}(x, y)=\frac{i}{\lambda} \iint U_{\mathrm{ref}}(X, Y) H_{\mathrm{det}}(X, Y) \frac{\exp (-i k|\vec{r}-\vec{R}|)}{|\vec{r}-\vec{R}|} d X d Y,
$$



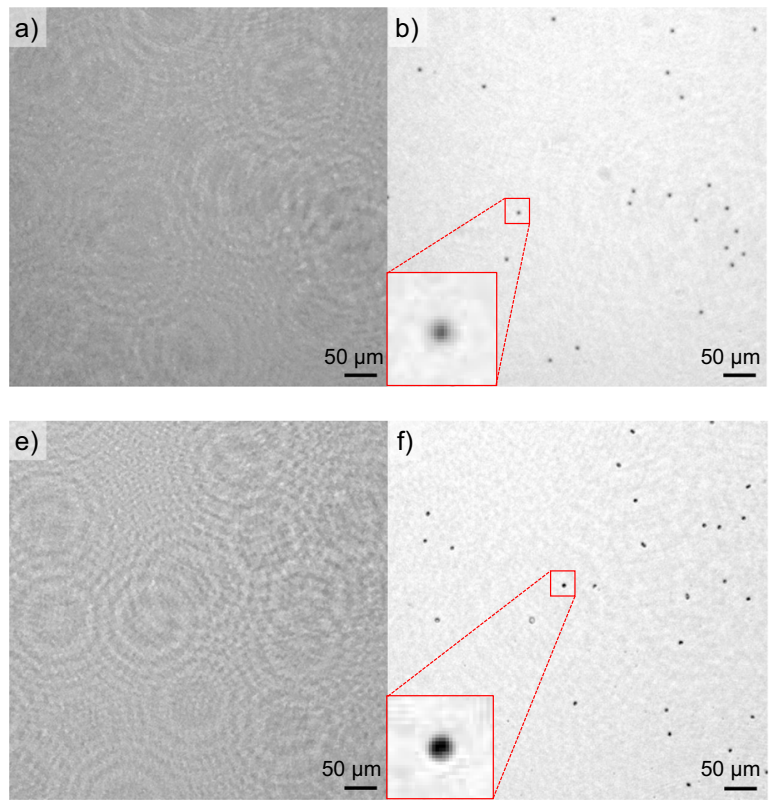
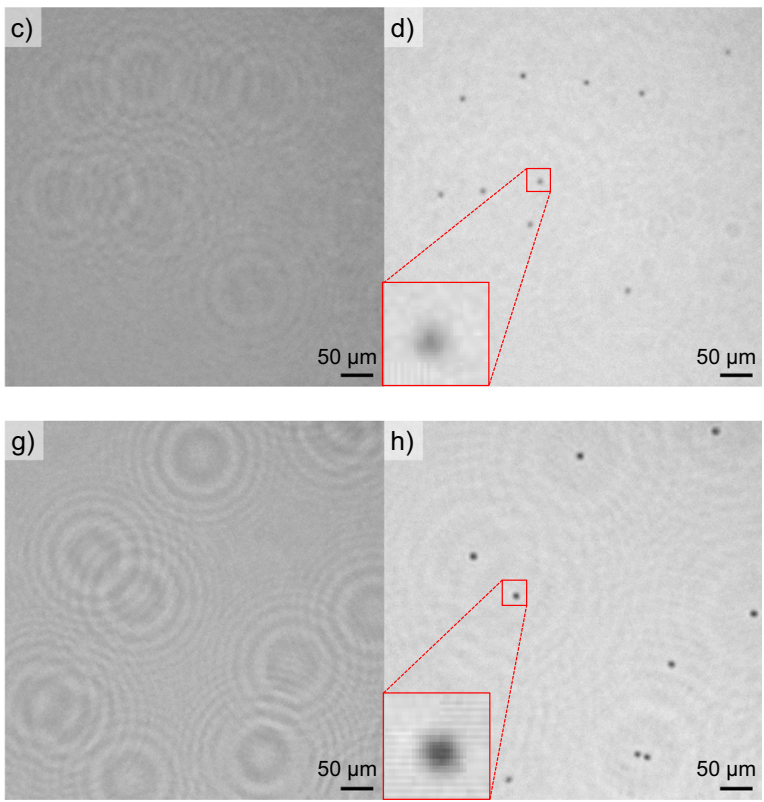

Figure 2. Holograms and according reconstructions of PMSs and human RBCs. Each inset shows a single sphere or RBC enlargd to five times its original size. (a) Hologram of PMSs captured with LD setup. (b) Reconstruction of (a). (c) Hologram of PMSs captured with LED setup. (d) Reconstruction of (c). (e) Hologram of RBCs captured with laser setup. (f) Reconstruction of (e). (g) Hologram of RBCs captured with LED setup. (h) Reconstruction of (g).

with the reference wave $U_{\text {ref }}(X, Y)$ and the intensity distribution of the hologram in the detector plane $H_{\text {det }}(X, Y)$. For the reconstruction, an open-source plugin for the open-source microscope software Fiji, see "Methods" section, that implements the angular spectrum estimation for small distances in the order of micrometers up to several centimeters, is employed. The amplitude distribution in the reconstructed plane is calculated using two Fourier transforms

$$
U_{\text {obj }}(z)=\mathcal{F}^{-1}\left\{\mathcal{F}\left\{U_{\text {det }}\right\} \exp \left(i z \sqrt{k^{2}-\frac{4 \pi^{2} n^{2}}{N p}}\right)\right\},
$$

where $z$ is the height of the reconstructed plane, $k$ the wave vector, $n$ the index of refraction, $N$ the number of pixels and $p$ the pixel size of the CMOS detector. $\mathcal{F}$ and $\mathcal{F}^{-1}$ denote the Fourier Transform and the inverse Fourier Transform. The formalism described above is implemented in two open-source reconstruction software packages, see "Methods" section. In the following, we elaborate and identify two easy to implement reconstruction software packages on a standard desktop computer or potentially also on a mobile phone. HoloPy, a software package for python, allows for hologram reconstruction, but also hologram simulation and scattering calculations. The algorithm to reconstruct point source holograms is based on Fresnel-Kirchhoff diffraction ${ }^{1}$. It considers a background subtracted hologram, experimental parameters including distances and light source wavelengths and then reconstructs the hologram using two Fourier transforms. Alternatively, hologram fitting is provided by HoloPy where the position of a scatterer is simulated in order to produce the same interference pattern instead of image back-propagation ${ }^{55}$. For the case of a known number of scatterers, this method can be recommended as it allows to reconstruct spherical or cylindrical object shapes. It is less practicable, however, when arbitrarily shaped micro-objects are of interest, as for example folded RBCs. For the latter case, an open-source plugin ${ }^{56}$ for Fiji, see "Methods" section, is a possible solution with a user-friendly graphical-user-interface, implemented in Java. Phase, amplitude and intensity distribution of micro-objects can be reconstructed at arbitrary heights in z-direction.

\section{Micro Particle and Cellular Imaging}

In the following, we first capture and image standardized PMSs of diameter $(6.5 \pm 0.2) \mu \mathrm{m}$ by both the LD and LED-based lens-less DIHM platform and reconstruct the resulting object properties by the Fiji plugin. Second, we investigate anonymized mature human RBCs as micro-objects in the same manner. Third, we image cell suspension culture TBY2s. The recorded holograms and subsequently reconstructed object planes for multiple PMSs and RBCs are depicted in Fig. 2. Laser-based DIHM hologram (a) and reconstruction (b) is presented next to LED-based DIHM hologram (c) and reconstruction results (d). Both insets depict an isolated PMS or RBCs enlarged to five times its original size. It becomes evident that the LD-based platform provides sharper images where a larger number of interference fringes can be captured per object. These fringes overlap within the hologram resulting in a hologram with more grainy texture as compared to the LED-based results in Fig. 2(a,c). In 

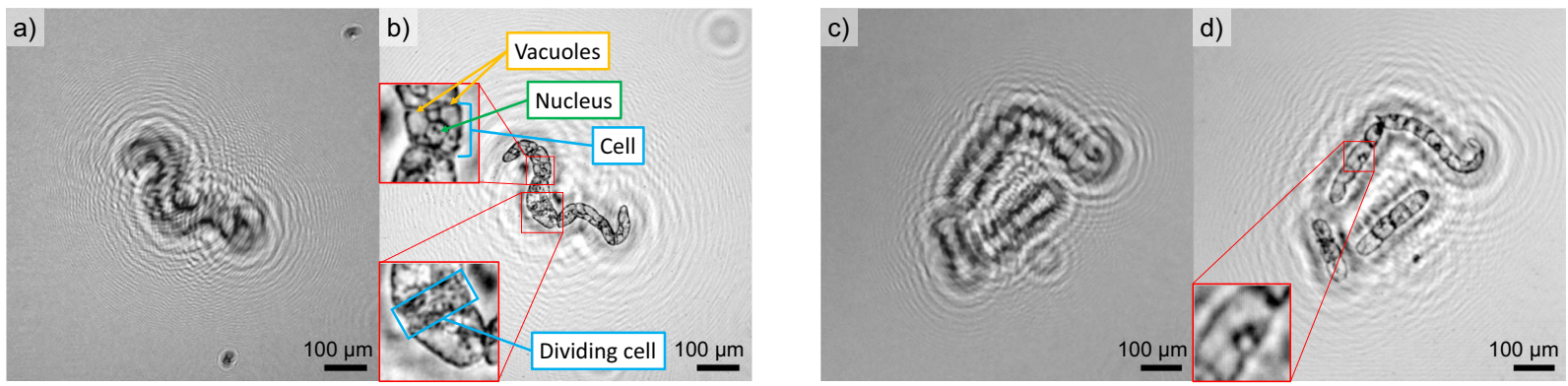

Figure 3. Holograms and according reconstructions of TBY2s. The insets are depicted magnified by a factor of three. (a) Hologram captured with LD setup. (b) Reconstruction of (a). (c) Hologram captured with LED setup. (d) Reconstruction of (c).

contrast, the LED-based reconstructed image is considerably more washed out resulting in comparably extended objects. Accordingly, for human RBCs imaged by the LD-based platform, the oval disk shape can clearly be resolved as depicted in Fig. 2(e,f). Several RBCs appear to be tilted in their spatial position, resulting in an elliptical shape. This is in stark contrast to the results obtained by the LED-based platform where information retrieval, for example on the cell morphology, are scarce. However, by the LED-based platform, individual cells can clearly be distinguished, thus exemplifying its potential for individual cell counting or tracking. In order to validate both platform's imaging capabilities also for extended cellular objects, fast growing plant tobacco TBY2s have been prepared and imaged. TBY2s are employed in various fields of plant biology as a model material and are ideally suited for cellular and molecular analyses ${ }^{57}$. Corresponding results are depicted in Fig. 3 . The recorded holograms and reconstructed object planes for an isolated TBY2s are depicted for LD-based DIHM hologram (a) and reconstruction (b) is presented whereas the hologram, obtained by the LED-based DIHM, is depicted in (c) and the corresponding reconstruction in (d). Both platforms allow to successfully access individual cell segments with a length of $50 \mu \mathrm{m}$ as well as internal structures including cell nuclei and vacuoles. In Fig. 3(b), a dividing cell undergoing mitosis can be observed. For LED illumination, individual vacuoles are not distinguishable. This is not surprising, as the vacuole membrane thickness is around one order of magnitude smaller than the cell wall thickness of (7-10) nm for plant vacuoles ${ }^{58}$ as compared to (71-87) nm for tobacco leaf cells walls ${ }^{59}$. However it is possible to identify the nuclei of several cells. Interestingly, TBY2s infer a more complex interference pattern as compared to both PMSs and RBCs, indicating a stronger absorption and thus increased hologram contrast. We found that Fiji revealed a substantially faster reconstruction as compared to HoloPy. The reconstruction of 10 planes of a digital hologram by the Fiji plugin demands 30 seconds computational time on a regular consumer PC as compared to several minutes by HoloPy. Towards larger volumes, the reconstruction time can theoretically be improved by performing computations on a graphics processing unit ${ }^{11}$ as demonstrated for live imaging ${ }^{60}$. In the following section, we aim to quantify the theoretical lateral resolution as well as the spatial resolution experimentally achieved by both the LD-based and LED-based DIHM platforms.

\section{Resolution}

In DIHM, the lateral resolution is bounded by the optical assembly numerical aperture $(N A)$ and the illumination wavelength $\lambda^{1}$ :

$$
\delta_{\text {lat }}=\frac{\lambda}{2 N A}
$$

suggesting a shorter wavelength for a higher lateral resolution. For a digital holographic microscope with a pixel number $N$, with pixel size $p$, an illumination wavelength $\lambda$ and a distance $s$ between object and detector plane, this translates to

$$
\delta_{\text {lat }}=\frac{s \lambda}{N p}
$$

As described $i^{61}$, a maximum lateral resolution can be achieved at a distance

$$
s_{\mathrm{opt}}=\frac{f}{1+\frac{f \lambda}{N p^{2}}}
$$

between object and detector, with the distance $f$ between light source and detector. This originates from the consideration, that a higher resolution is possible, the closer the object is placed to the detector, however at the same time the interference fringes move closer. Here, $s_{\mathrm{opt}}$ denotes the distance at which different interference rings are still resolved by different camera pixels. The axial resolution of the system can be calculated according to

$$
\delta_{\mathrm{ax}}=\frac{\lambda}{2(N A)^{2}}=\frac{2 s^{2} \lambda}{(N p)^{2}} .
$$




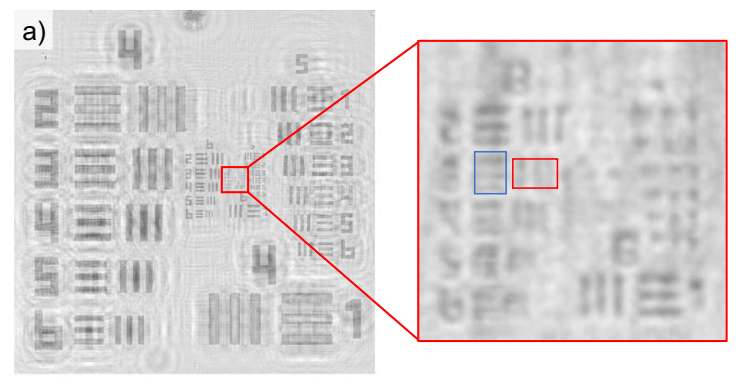

c)

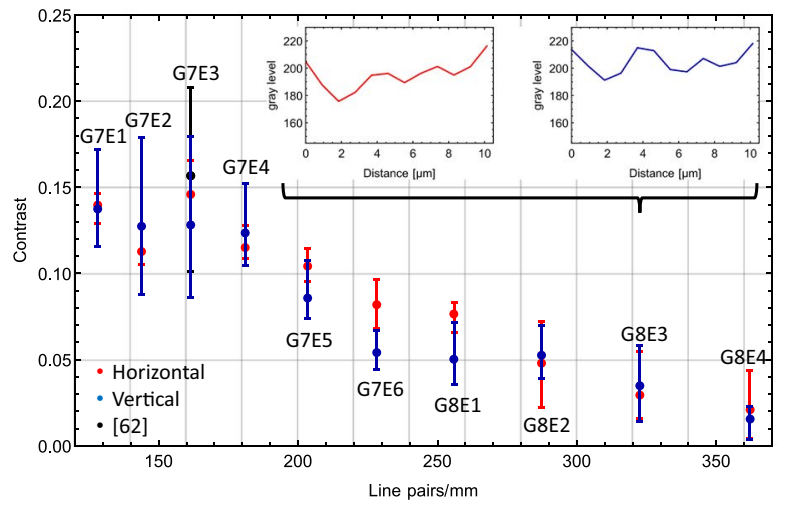

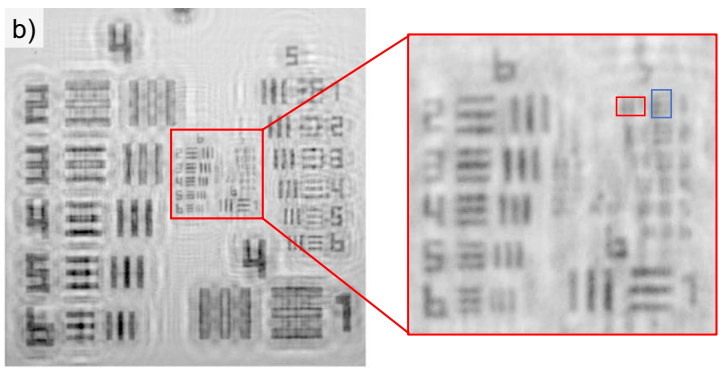

d)

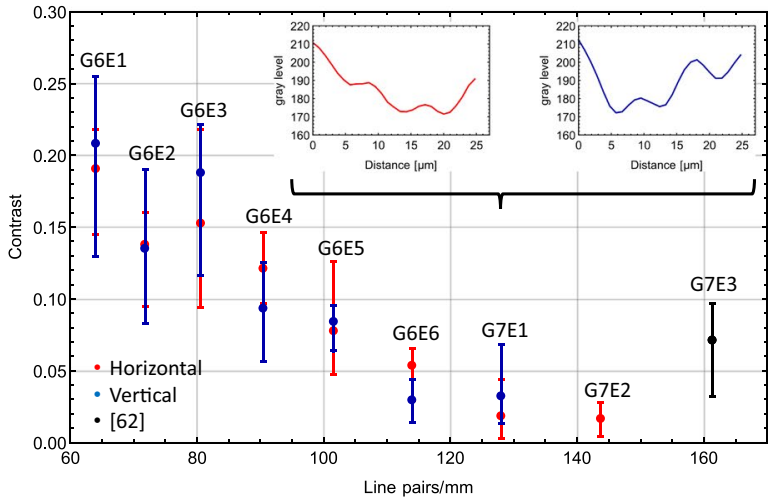

Figure 4. Imaging of a 1951 USAF target to quantify the accessible spatial resolution. (a) LD setup. Groups 8 and 9 are shown enlarged. (b) LED setup. Groups 6 to 9 are shown enlarged. (c) Contrast of the different elements for LD illumination. The inset shows the intensity profile plot for horizontal and vertical elements 3 of group 8. (d) Contrast for LED illumination. Additionally included are reported contrast values ${ }^{62}$, where either an unspecified laser or an LED emitting at $470 \mathrm{~nm}$ ( $25 \mu \mathrm{m}$ pinhole) had been employed (not specified further).

By equation (6), the theoretical resolution for both developed platforms can be estimated. For $p=1.12 \mu \mathrm{m}$, $N=2464, f=30 \mathrm{~mm}, \lambda_{\mathrm{LED}}=430 \mathrm{~nm}$ and $\lambda_{\mathrm{LD}}=405 \mathrm{~nm}$, respectively, lateral resolutions of $\delta_{\mathrm{LED}}=0.92 \mu \mathrm{m}$ and $\delta_{\mathrm{LD}}=0.87 \mu \mathrm{m}$ are theoretically possible by the selected, optimum platform design. To evaluate the experimentally achieved resolution, a 1951 United States Air Force (USAF) microscopic imaging test target on a glass microscopic slide serves as a reference object consisting of groups of horizontal and vertical lines with decreasing spatial frequency. The resulting reconstructed amplitude images acquired with the LED (a) and LD (b) setup are depicted in Fig. 4. With LED illumination, element 1 of group 7 is the last resolvable element corresponding to a resolution of 128 line pairs $/ \mathrm{mm}$ and a line width of $3.91 \mu \mathrm{m}$. For LD illumination, element 3 of group 8 is still resolvable, leading to a resolution of 322.5 line pairs $/ \mathrm{mm}$ and a line width of $1.55 \mu \mathrm{m}$. This is mostly a result of the higher temporal and spatial resolution of the laser in comparison to the used LED, as well as the smaller wavelength. The resolving power with $\mathrm{LD}$ illumination is thus significantly higher.

Finally, to quantify the achieved image contrast and thus evaluate the capability of the developed DIHM setups, in the following the intensity of a single USAF element is averaged along its axis and plotted (see red and blue rectangle in Fig. 4(a,b). Then, the contrast of consecutive extreme points is calculated by $K=\frac{I_{\max }-I_{\min }}{I_{\max }+I_{\min }}$. Each element consists of 5 dark lines which leads to 4 contrast values. The average contrast is then displayed in dependence on the resolution in line pairs $/ \mathrm{mm}$, with the error bars ranging from the minimum to the maximum value. The resulting image contrast of different elements of the respective USAF image is depicted in Fig. 4(c,d). Results obtained by both light sources indicate decreasing contrast values with increasing line pairs. The maximum resolvable elements are as shown by the the intensity profile plots for horizontal and vertical elements 3 of group 8 in the inset of Fig. 4(c). For LED, the maximum resolvable element is element 1 of group 7 as depicted in the inset of Fig. 4(d). We compared our contrast values to reported values ${ }^{62}$, where an unspecified laser or a LED with a central wavelength of $470 \mathrm{~nm}(25 \mu \mathrm{m}$ pinhole) was used for image acquisition. The reported value for laser illumination coincides with our results. With LED illumination, the reported value reaches a higher resolution, group 7 element 3 is still resolvable. This could be due to a higher signal-to-noise ratio of the employed camera, the smaller bandwidth of only $10 \mathrm{~nm}$ as well as the use of advanced reconstruction algorithms. The achieved spatial resolution of both setups is suited to image and detect individual micro-particles and cellular objects including ensembles of microscopic biological samples. For the LD-based DIHM, sharper interference pattern and a higher number of interference fringes could be captured. Thus, more object information is retrieved yielding a crisper reconstructed image including more details. We attribute this to the LD's higher second-order temporal coherence as well as spatial coherence. The LED's spatial coherence could be increased by reducing the pinhole diameter which would require increased LED biasing currents which then require efficient cooling of the LED and thereby increasing the physical dimensions of the setup. Our DIHMs allow to image cellular objects with dimensions smaller than $10 \mu \mathrm{m}$ as well as microscopy of larger objects with a spatial resolution of $3.91 \mu \mathrm{m}$. To 
ensure maximum possible resolution, a LD-based DIHMs is recommended, whereas for applications where high temporal stability, compact set-up, ruggedness and reduced costs a required, LED-based DIHMs is recommended.

\section{Conclusion}

A 3D printable platform for lens-less holographic cellular imaging with open accessible software solutions has been developed delivering spatial resolutions of $1.55 \mu \mathrm{m}$ by LD or $3.91 \mu \mathrm{m}$ by LED illumination. A $405 \mathrm{~nm}$ Blu-ray semiconductor laser-pickup coupled to an optical fibre and a $430 \mathrm{~nm}$ high power LED in combination with a $15 \mu \mathrm{m}$ pinhole have been successfully employed as DIHM light sources. Despite its lower degree of temporal coherence, the LED proved to be of advance in terms of implementation, price and lower safety concerns. A single-board portable Raspberry Pi computer and camera operate the light sources as well as perform the image acquisition. By an open-source software implementation of the Fresnel-Kirchhoff algorithm, we imaged and successfully reconstructed $6.5 \mu \mathrm{m}$ PMS and human RBCs with a diameter of about $8 \mu \mathrm{m}$, as well as TBY2s with an individual size of about $50 \mu \mathrm{m}$. Less than $1.1 \mu \mathrm{W}$ of optical power were sufficient for holographic imaging microscopy. Such ultra-low optical power can be of critical importance for live cell imaging where light exposure of the cells needs to be as low as possible. Equally compact setups could be envisioned for the LD when fibre-coupled LDs are available, which are however considerably expensive. The DIHM setup presented here may serve as a reliable, easy to implement and flexible to extend solution for student an early researcher education and for different demands in microscopic imaging. The total costs for the LED setup amount to \$190 (\$3 LED, \$75 Pinhole, \$35 Raspberry Pi 3, \$25 Raspberry Pi Cam v2, \$25 3D print, \$27 power bank) and thus enables a convenient entry into the wide field of digital holography. Future work could include the integration of the DIHM with micro-fluidic channels ${ }^{48,63,64}$ or considering machine learning algorithms to automatically count and identify particles $^{65}$, as well as diagnose illnesses such as meningitis ${ }^{66}$, iron-deficiency anemia or diabetes mellitus ${ }^{67}$. The developed 3D-printable photonic platform might help facilitating reproducibility of results obtained in different laboratories and prototyping of specific improvements and advancements of the DIHM setups. The cost-efficient open science and open hard and software platform aims in particular at contributing towards a democratization of scientific knowledge ${ }^{68}$.

\section{Methods}

DIHM construction, light sources, opto-electronics, electrical circuits and 3D print. The Blu-ray LD-pickup (SF-AW210) has been dismounted from a commercially available standard computer Blu-ray optical head and its emits a maximum optical output power of $300 \mathrm{~mW}$ at a wavelength of $405 \mathrm{~nm}$ for an injection current of $150 \mathrm{~mW}$. The LD-pickup beam is collimated and focussed by two aspheric lenses with an effective focal length of $11 \mathrm{~mm}$ ( $\$ 87$ each, C220TMD-A, Thorlabs Inc.) into a $2 \mathrm{~m}$ long silica core FC/PC single-mode optical fibre with a core diameter of $3 \mu \mathrm{m}(\$ 99$, S405-XP, Nufern Inc.) the coupling is adjust to ensure a low laser power of $24.5 \mu \mathrm{W}$ at the fibre exit which proved optimum for DIHM imaging. The emitted divergent beam (Rayleigh length $z_{\mathrm{r}}=21 \mu \mathrm{m}$ ) has $\mathrm{TEM}_{00}$ intensity profile and a mode field diameter of $2 w_{0}=3.3 \mu \mathrm{m}$. Within the plane of the object glass plate, at $z=5.91 \mathrm{~mm}$, the divergent beam impinges the objects under investigation with a power density of 4.2 Watt $/ \mathrm{mm}^{2}$ and with a beam diameter of $3.8 \mathrm{~mm}\left(1 / \mathrm{e}^{2}\right)$. In the CMOS detector plane $(f=30 \mathrm{~mm})$, the beam diameter amounts to $4.7 \mathrm{~mm}\left(1 / \mathrm{e}^{2}\right)$. The high-power LED (\$3, $3 \mathrm{~W}$ High Power LED $430 \mathrm{~nm}-435 \mathrm{~nm}$ hyper violet, Avonec, Germany ${ }^{69}$ ) emits at wavelengths centered at around $430 \mathrm{~nm}$ with spectral bandwidth of $\Delta \lambda_{\mathrm{LED}}$ of $15 \mathrm{~nm}$ (full-width-half-maximum) corresponding to a coherence length of $L_{c, L E D}=\lambda_{\text {LED }}^{2} \times\left(\pi \times \Delta \lambda_{\text {LED }}\right)^{-1}=3.9 \mu \mathrm{m}$ for a Gaussian intensity distribution, with $\Delta \lambda$ the full width at half-maximum spectral line width ${ }^{70}$. A high-precision stainless steel pinhole with a diameter of $(15 \pm 1.5) \mu \mathrm{m}(\$ 75, \mathrm{P} 15 \mathrm{D}$, Thorlabs Inc.) has been employed as all $3 \mathrm{D}$ printing attempts yet did not yield a necessary sufficiently high mechanical grade circular diameter. All neccessary parts for the LED setup can be found at https://github.com/teph12/DIHM. All parts and Raspberry Pi housing were printed with a commercial 3D-printer (\$880, Prusa i3 MK3, Prusa Research s.r.o.) using standard polylactide synthetic polymer filament with $1.75 \mathrm{~mm}$ diameter. A spatial printing resolution of $0.4 \mathrm{~mm}$, parallel to the optical table, and $0.05 \mathrm{~mm}$, in vertical or z-direction is available. Within the printed DIHM housing, LED and pinhole were fixed with tape (\$9, 3M-ID 70005241826 Scotch Magic Tape, 3M Inc.). The case with VESA mounts for Raspberry Pi $3(\mathrm{~B} / \mathrm{B}+)$, Pi $2 \mathrm{~B}$, and Pi $1 \mathrm{~B}+$ can be accessed by ${ }^{71}$. The CMOS camera module is glued to the upper part of the box after removing the lens mounted in front of the module. We observed that otherwise strong hologram distortions appeared. In order to electrically bias the LD, a commercial LD driver was used to provide a constant output current. However, an open-source driver is under construction while all parts are available for in total $\$ 20^{72}$. For the LED, a custom soldered circuit has been developed accessing Raspberry Pi's general purpose input/output (GPIO). The assembled system is depicted in $5 \mathrm{~b}$ ). The driver circuits can well be integrated into the 3D printed Raspberry Pi housing. A diagram of the circuit can be seen in Fig. 5(a). The portable computer, camera and DIHM light sources are supplied with electrical power by a conventional power bank battery pack with maximum output power of $10 \mathrm{~W}$ (\$27, Aukey PB-N36). A computer monitor and computer mouse are required for the hologram acquisition. V_BATTERY is the voltage provided by the power bank, whereas V_ TRIGGER is the voltage between the Raspberry Pi GPIO and ground. It triggers a transistor (BD135), which lets a current of $125 \mathrm{~mA}$ flow through the LED.

Light source biasing, image detection and aquisition. A portable single-board computer and camera module with a $3280 \times 2464$ pixel CMOS chip and pixel dimensions of $(1.12 \times 1.12) \mu \mathrm{m}^{2}$, sensor size $(3.674 \times 2.760) \mathrm{mm}^{2}\left(\$ 35\right.$, Raspberry Pi 3 with $\$ 25$ Raspberry Pi Camera v2, Raspberry Pi Foundation, UK $\left.{ }^{51}\right)$ serve as light source driver and hologram acquisition. 


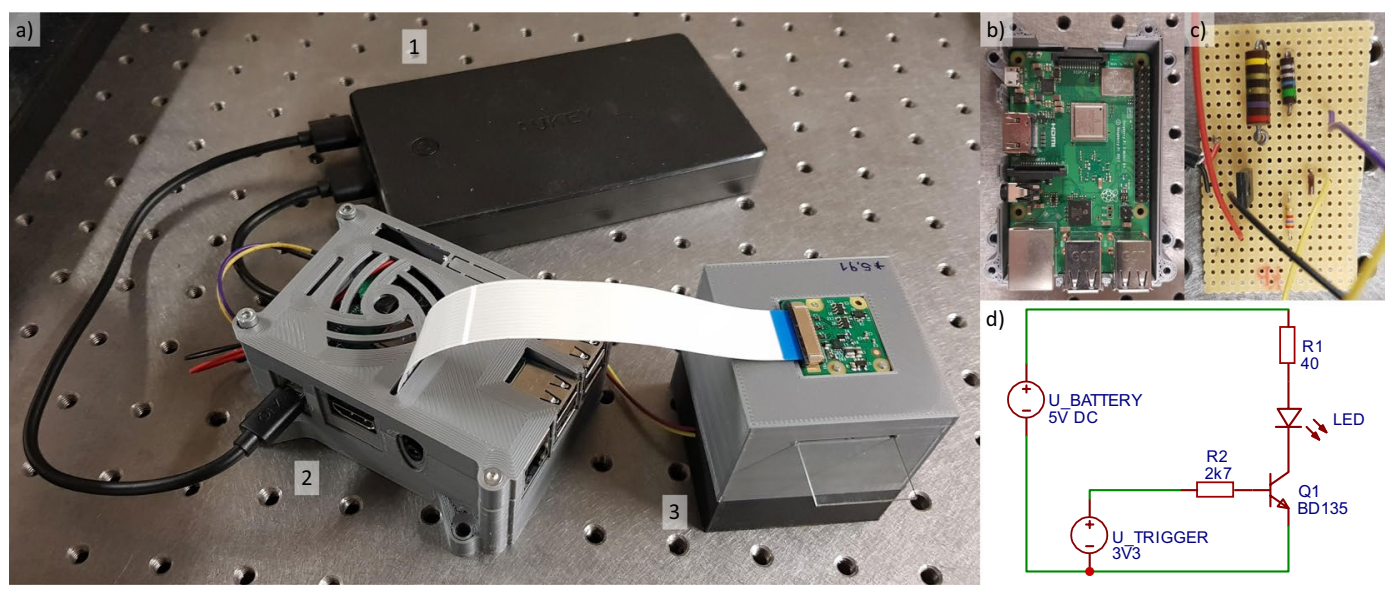

Figure 5. (a) The fully portable DIHM system consisting of (1) USB-port power bank, (2) 3D printed housing containing Raspberry Pi and LED control circuit, (3) LED DIHM system as presented in Fig. 1(d). (b) Closeup of the Raspberry Pi single-board computer. (c) Closeup of the LED control circuit. (d) Schematic of the circuit for biasing the LED using the Raspberry Pi’s GPIOs.

Sample preparation. The RBC samples under investigation are standard anonymized human RBC reference cells (\$27, A1A2B0 $4 \times 10$, Immucor Med. Diagnostik GmbH, Germany). They are diluted in saline solution to a high degree (up to $0.02 \%$ ). The employed polystyrene micro-spheres ( $\$ 129$, BS-Partikel GmbH, Germany) have a diameter of $(6.5 \pm 0.2) \mu \mathrm{m}$. They are equally high diluted in distilled water. Furthermore a few ml of ordinary dish detergent are added to prevent aggregation and adhesion of the micro-spheres. After diluting the particular object a drop of the solution is placed by a standard plastic pasteur pipette on a high transmission flat glass microscope slide $(75 \mathrm{~mm} \times 25 \mathrm{~mm} \times 1 \mathrm{~mm}, \mathrm{~B} 270 \mathrm{I}$, SCHOTT AG) and then covered with a glass cover plate $\left(22 \mathrm{~mm} \times 22 \mathrm{~mm} \times 0.15 \mathrm{~mm}\right.$, B270 I, SCHOTT AG). Nicotiana tabacum cv. BY-2 suspension plant cell cultures ${ }^{73}$ were grown in liquid saline medium based on a modified Linsmaier and Skoog medium with agitation on a incubator shaker. The cells have been grown in $50 \mathrm{ml}$ medium within a $250 \mathrm{ml}$ glass flask. Every 7 days, $5 \%$ inoculum had been transferred into a fresh medium ${ }^{74,75}$ and stored permanently on an incubator shaker.

Image acquisition, reconstruction and resolution validation. The pictures are acquired with a fixed white balance. For details see the camera file on https://github.com/teph12/DIHM. The image is then transferred to a PC with Windows 10 operating system and equipped with an Intel i3 processor and 8 GB of RAM, with Fiji installed. In Fiji the image is converted to a 32-bit black and white picture, which is then loaded in the Numerical Reconstruction plugin. Here, using the parameters of image acquisition (distance between camera and object, wavelength, image size) the image is reconstructed. Subsequently the image contrast is normalized and enhanced by $0.2 \%$. For the quantification of the experimentally achieved spatial resolution, a commercially available standardized 1951 USAF positive high-contrast chrome on quartz glass microscopic imaging test target created by photo lithography on a glass microscopic slide serves as a reference object ( $\$ 900$, Ready Optics, US). It consists of groups of horizontal and vertical lines with standardized spatial frequencies starting at group 4, element 1 with $31 \mu \mathrm{m}$ spacing and ending at group 11, element 6 with $137 \mathrm{~nm}$ spacing.

\section{References}

1. Jericho, M. H. \& Kreuzer, H. J. Point source digital in-line holographic microscopy. In Coherent Light Microscopy, 3-30, https://doi. org/10.1007/978-3-642-15813-1_1 (Springer Berlin Heidelberg, 2010).

2. Gabor, D. A new microscopic principle. Nature 161, 777-778, https://doi.org/10.1038/161777a0 (1948).

3. Rong, L. et al. Terahertz in-line digital holography of human hepatocellular carcinoma tissue. Sci. Reports 5, https://doi.org/10.1038/ srep08445 (2015).

4. Repetto, L., Chittofrati, R., Piano, E. \& Pontiggia, C. Infrared lensless holographic microscope with a vidicon camera for inspection of metallic evaporations on silicon wafers. Opt. Commun. 251, 44-50, https://doi.org/10.1016/j.optcom.2005.02.061 (2005).

5. Faridian, A. et al. Nanoscale imaging using deep ultraviolet digital holographic microscopy. Opt. Express 18, 14159, https://doi. org/10.1364/oe.18.014159(2010).

6. Daloglu, M. U. et al. Computational on-chip imaging of nanoparticles and biomolecules using ultraviolet light. Sci. Reports 7, https:// doi.org/10.1038/srep44157 (2017)

7. Krenkel, M., Toepperwien, M., Alves, F. \& Salditt, T. Three-dimensional single-cell imaging with x-ray waveguides in the holographic regime. Acta Crystallogr. Sect. A Foundations Adv. 73, 282-292, https://doi.org/10.1107/s2053273317007902 (2017).

8. Gorkhover, T. et al. Femtosecond x-ray fourier holography imaging of free-flying nanoparticles. Nat. Photonics 12, 150-153, https:// doi.org/10.1038/s41566-018-0110-y (2018).

9. Kreuzer, H. Low energy electron point source microscopy. Micron 26, 503-509, https://doi.org/10.1016/0968-4328(95)00021-6 (1995).

10. Lichte, H. \& Lehmann, M. Electron holography-basics and applications. Reports on Prog. Phys. 71, 016102, https://doi. org/10.1088/0034-4885/71/1/016102 (2007).

11. Hobson, P. R., Nebrensky, J. J. \& Reid, I. D. Challenges in using GPUs for the real-time reconstruction of digital hologram images. J. Physics: Conf. Ser. 415, 012042, https://doi.org/10.1088/1742-6596/415/1/012042 (2013).

12. Molaei, M. \& Sheng, J. Imaging bacterial 3D motion using digital in-line holographic microscopy and correlation-based de-noising algorithm. Opt. Express 22, 32119, https://doi.org/10.1364/oe.22.032119 (2014). 
13. Carl, D., Kemper, B., Wernicke, G. \& von Bally, G. Parameter-optimized digital holographic microscope for high-resolution livingcell analysis. Appl. Opt. 43, 6536, https://doi.org/10.1364/ao.43.006536 (2004).

14. Giuliano, C. B., Zhang, R. \& Wilson, L. G. Digital inline holographic microscopy (DIHM) of weakly-scattering subjects. J. Vis. Exp. https://doi.org/10.3791/50488 (2014).

15. Sanz, M., Picazo-Bueno, J. Á., Granero, L., García, J. \& Micó, V. Compact, cost-effective and field-portable microscope prototype based on MISHELF microscopy. Sci. Reports 7, 43291, https://doi.org/10.1038/srep43291 (2017).

16. Rostykus, M. \& Moser, C. Compact lensless off-axis transmission digital holographic microscope. Opt. Express 25, 16652, https:// doi.org/10.1364/oe.25.016652 (2017).

17. Mudanyali, O., Oztoprak, C., Tseng, D., Erlinger, A. \& Ozcan, A. Detection of waterborne parasites using field-portable and costeffective lensfree microscopy. Lab on a Chip 10, 2419, https://doi.org/10.1039/c004829a (2010).

18. Tseng, D. et al. Lensfree microscopy on a cellphone. Lab on a Chip 10, 1787, https://doi.org/10.1039/c003477k (2010).

19. Greenbaum, A. \& Ozcan, A. Maskless imaging of dense samples using pixel super-resolution based multi-height lensfree on-chip microscopy. Opt. Express 20, 3129, https://doi.org/10.1364/oe.20.003129 (2012).

20. Luo, W., Zhang, Y., Feizi, A., Göröcs, Z. \& Ozcan, A. Pixel super-resolution using wavelength scanning. Light. Sci. \& Appl. 5, e16060-e16060, https://doi.org/10.1038/lsa.2016.60 (2015).

21. Vandewiele, S., Strubbe, F., Schreuer, C., Neyts, K. \& Beunis, F. Low coherence digital holography microscopy based on the LorenzMie scattering model. Opt. Express 25, 25853, https://doi.org/10.1364/oe.25.025853 (2017).

22. Feng, S. \& Wu, J. Differential holographic reconstruction for lensless in-line holographic microscope with ultra-broadband light source illumination. Opt. Commun. 430, 9-13, https://doi.org/10.1016/j.optcom.2018.08.033 (2019).

23. Serabyn, E., Liewer, K., Lindensmith, C., Wallace, K. \& Nadeau, J. Compact, lensless digital holographic microscope for remote microbiology. Opt. Express 24, 28540, https://doi.org/10.1364/oe.24.028540 (2016).

24. Leith, E. N. \& Upatnieks, J. Reconstructed wavefronts and communication theory. J. Opt. Soc. Am. 52, 1123, https://doi.org/10.1364/ josa.52.001123 (1962).

25. Langehanenberg, P., von Bally, G. \& Kemper, B. Application of partially coherent light in live cell imaging with digital holographic microscopy. J. Mod. Opt. 57, 709-717, https://doi.org/10.1080/09500341003605411 (2010).

26. Ravaro, M. et al. Mid-infrared digital holography and holographic interferometry with a tunable quantum cascade laser. Opt. Lett. 39, 4843, https://doi.org/10.1364/ol.39.004843 (2014).

27. Kim, M. K. Principles and techniques of digital holographic microscopy. J. Photonics for Energy 018005, https://doi.org/10.1117/6.0000006 (2010).

28. Latychevskaia, T. \& Fink, H.-W. Solution to the twin image problem in holography. Phys. Rev. Lett. 98, https://doi.org/10.1103/ physrevlett.98.233901 (2007).

29. Chen, B. K., Chen, T.-Y., Hung, S. G., Huang, S.-L. \& Lin, J.-Y. Twin image removal in digital in-line holography based on iterative inter-projections. J. Opt. 18, 065602, https://doi.org/10.1088/2040-8978/18/6/065602 (2016)

30. Denis, L., Fournier, C., Fournel, T. \& Ducottet, C. Numerical suppression of the twin image in in-line holography of a volume of micro-objects. Meas. Sci. Technol. 19, 074004, https://doi.org/10.1088/0957-0233/19/7/074004 (2008).

31. Greenbaum, A., Feizi, A., Akbari, N. \& Ozcan, A. Wide-field computational color imaging using pixel super-resolved on-chip microscopy. Opt. Express 21, 12469, https://doi.org/10.1364/oe.21.012469 (2013).

32. Frentz, Z., Kuehn, S., Hekstra, D. \& Leibler, S. Microbial population dynamics by digital in-line holographic microscopy. Rev. Sci. Instruments 81, 084301, https://doi.org/10.1063/1.3473937 (2010).

33. Bishara, W., Su, T.-W., Coskun, A. F. \& Ozcan, A. Lensfree on-chip microscopy over a wide field-of-view using pixel superresolution. Opt. Express 18, 11181, https://doi.org/10.1364/oe.18.011181 (2010).

34. Bishara, W., Zhu, H. \& Ozcan, A. Holographic opto-fluidic microscopy. Opt. Express 18, 27499, https://doi.org/10.1364/oe.18.027499 (2010).

35. Zhu, H., Isikman, S. O., Mudanyali, O., Greenbaum, A. \& Ozcan, A. Optical imaging techniques for point-of-care diagnostics. Lab Chip 13, 51-67, https://doi.org/10.1039/c2lc40864c (2013).

36. LyncéeTec. Digital holographic microscopes. https://www.lynceetec.com/ (2019).

37. Jericho, S. K., Garcia-Sucerquia, J., Xu, W., Jericho, M. H. \& Kreuzer, H. J. Submersible digital in-line holographic microscope. Rev. Sci. Instruments 77, 043706, https://doi.org/10.1063/1.2193827 (2006).

38. 4-Deep. Holographic \& fluorescence microscopes. http://4-deep.com/ (2019).

39. Fraunhofer IPM. HoloTop - High-precision inline 3D measurement. https://www.ipm.fraunhofer.de/en/bu/production-controlinline-measurement-techniques/applications/holotop-holographic-3d-object-measurement.html (2019).

40. Finkeldey, M., Göring, L., Brenner, C., Hofmann, M. \& Gerhardt, N. C. Depth-filtering in common-path digital holographic microscopy. Opt. Express 25, 19398, https://doi.org/10.1364/oe.25.019398 (2017).

41. Park, J. H., Go, T. \& Lee, S. J. Label-free sensing and classification of old stored blood. Annals Biomed. Eng. 45, 2563-2573, https:// doi.org/10.1007/s10439-017-1902-9 (2017).

42. Rappaz, B. et al. Spatial analysis of erythrocyte membrane fluctuations by digital holographic microscopy. Blood Cells, Mol. Dis. 42, 228-232, https://doi.org/10.1016/j.bcmd.2009.01.018 (2009).

43. Zakrisson, J., Schedin, S. \& Andersson, M. Cell shape identification using digital holographic microscopy. Appl. Opt. 54, 7442, https://doi.org/10.1364/ao.54.007442 (2015).

44. Park, H. et al. Characterizations of individual mouse red blood cells parasitized by babesia microti using 3-D holographic microscopy. Sci. Reports 5, https://doi.org/10.1038/srep10827 (2015).

45. Kastl, L., Isbach, M., Dirksen, D., Schnekenburger, J. \& Kemper, B. Quantitative phase imaging for cell culture quality control. Cytom. Part A 91, 470-481, https://doi.org/10.1002/cyto.a.23082 (2017).

46. El-Schich, Z., Mölder, A. L. \& Wingren, A. G. Quantitative phase imaging for label-free analysis of cancer cells-focus on digital holographic microscopy. Appl. Sci. 8, 1027, https://doi.org/10.3390/app8071027 (2018).

47. Merola, F. et al. Searching and identifying microplastics in marine environment by digital holography. The Eur. Phys. J. Plus 133, https://doi.org/10.1140/epjp/i2018-12190-y (2018).

48. Choi, Y.-S. \& Lee, S.-J. Three-dimensional volumetric measurement of red blood cell motion using digital holographic microscopy. Appl. Opt. 48, 2983, https://doi.org/10.1364/ao.48.002983 (2009).

49. Dimiduk, T. G. et al. A simple, inexpensive holographic microscope. In Biomedical Optics and 3-D Imaging, https://doi.org/10.1364/ biomed.2010.jma38 (OSA, 2010).

50. Shimobaba, T., Taniguchi, Y., Shiraki, A., Masuda, N. \& Ito, T. Portable and low-cost digital holographic microscopy using web camera, point light source LED and open-source libraries. In Biomedical Optics and 3-D Imaging, https://doi.org/10.1364/ biomed.2012.jm3a.50 (OSA, 2012).

51. Raspberry Pi Foundation. Raspberry pi - teach, learn, and make with raspberry pi. https://www.raspberrypi.org/ (2019).

52. Manoharan Lab, Harvard University. HoloPy: Holography and Light Scattering in Python. https://holopy.readthedocs.io/en/3.1.1/ index.html (2016).

53. Schindelin, J. et al. Fiji: an open-source platform for biological-image analysis. Nat. Methods 9, 676-682, https://doi.org/10.1038/ nmeth.2019 (2012).

54. Latychevskaia, T. \& Fink, H.-W. Practical algorithms for simulation and reconstruction of digital in-line holograms. Appl. Opt. 54, 2424, https://doi.org/10.1364/ao.54.002424 (2015). 
55. Dimiduk, T. G., Perry, R. W., Fung, J. \& Manoharan, V. N. Random-subset fitting of digital holograms for fast threedimensional particle tracking [invited]. Appl. Opt. 53, G177, https://doi.org/10.1364/ao.53.00g177 (2014).

56. Garcia-Sucerquia, J. Multispectral digital lensless holographic microscopy: from femtosecond laser to white light LED. J. Physics: Conf. Ser. 605, 012011, https://doi.org/10.1088/1742-6596/605/1/012011 (2015).

57. Yokoyama, R., Tanaka, D., Fujino, T., Itoh, T. \& Nishitani, K. Cell wall dynamics in tobacco BY-2 cells. In Tobacco BY-2 Cells, 217-230, https://doi.org/10.1007/978-3-662-10572-6_15 (Springer Berlin Heidelberg, 2004).

58. Esau, K. \& Cheadle, V. I. An evaluation of studies on ultrastructure of tonoplast in sieve elements. Proc. Natl. Acad. Sci. 48, 1-8, https://doi.org/10.1073/pnas.48.1.1 (1962).

59. Hoffmann-Benning, S., Willmitzer, L. \& Fisahn, J. Analysis of growth, composition and thickness of the cell walls of transgenic tobacco plants expressing a yeast-derived invertase. Protoplasma 200, 146-153, https://doi.org/10.1007/bf01283290 (1997).

60. Locatelli, M. et al. Real-time terahertz digital holography with a quantum cascade laser. Sci. Reports 5, https://doi.org/10.1038/ srep13566 (2015).

61. Serabyn, E., Liewer, K. \& Wallace, J. K. Resolution optimization of an off-axis lensless digital holographic microscope. Appl. Opt. 57, A172, https://doi.org/10.1364/ao.57.00a172 (2017).

62. Feng, S. \& Wu, J. Resolution enhancement method for lensless in-line holographic microscope with spatially-extended light source. Opt. Express 25, 24735, https://doi.org/10.1364/oe.25.024735 (2017).

63. Cheong, F. C. et al. Holographic characterization of colloidal particles in turbid media. Appl. Phys. Lett. 111, 153702, https://doi. org/10.1063/1.4999101 (2017).

64. Bianco, V. et al. Endowing a plain fluidic chip with micro-optics: a holographic microscope slide. Light. Sci. \& Appl. 6, e17055, https://doi.org/10.1038/lsa.2017.55 (2017).

65. Roy, M., Jin, G., Seo, D., Nam, M.-H. \& Seo, S. A simple and low-cost device performing blood cell counting based on lens-free shadow imaging technique. Sensors Actuators B: Chem. 201, 321-328, https://doi.org/10.1016/j.snb.2014.05.011 (2014).

66. Delacroix, R. et al. Cerebrospinal fluid lens-free microscopy: a new tool for the laboratory diagnosis of meningitis. Sci. Reports 7 , https://doi.org/10.1038/srep39893 (2017).

67. Kim, G., Jo, Y., Cho, H., Seok Min, H. \& Park, Y. Learning-based screening of hematologic disorders using quantitative phase imaging of individual red blood cells. Biosens. Bioelectron. 123, 69-76, https://doi.org/10.1016/j.bios.2018.09.068 (2019).

68. Baden, T. et al. Open labware: 3-D printing your own lab equipment. PLOS Biol. 13, e1002086, https://doi.org/10.1371/journal. pbio.1002086 (2015).

69. Avonec. https://avonec.de/ (2018)

70. Leonard Mandel, E. W. Optical Coherence and Quantum Optics (Cambridge University Press, 1995).

71. 0110-M-P0. Raspberry Pi 3 (B/B+), Pi 2 B, and Pi 1 B + case with VESA mounts. https://www.thingiverse.com/thing:922740 (2015).

72. Innolasers. https://innolasers.com/ (2013)

73. Nagata, T., Nemoto, Y. \& Hasezawa, S. Tobacco BY-2 cell line as the "HeLa" cell in the cell biology of higher plants. In International Review of Cytology, 1-30, https://doi.org/10.1016/s0074-7696(08)62452-3 (Elsevier, 1992).

74. Nocarova, E. \& Fischer, L. Cloning of transgenic tobacco by-2 cells; an efficient method to analyse and reduce high natural heterogeneity of transgene expression. BMC Plant Biol. 9, 44, https://doi.org/10.1186/1471-2229-9-44 (2009).

75. Mercx, S., Tollet, J., Magy, B., Navarre, C. \& Boutry, M. Gene inactivation by crispr-cas9 in nicotiana tabacum by-2 suspension cells. Front. Plant Sci. 7, 40, https://doi.org/10.3389/fpls.2016.00040 (2016)

\section{Acknowledgements}

The authors thank Markus Langhans for assistance with cell cultivation and preparation, Christoph Weber for 3D printing the Raspberry Pi housing, Alexander Rohrbach for helpful discussions as well as W. Elsäßer for support. S.B. acknowledges support by the German Research Foundation (DFG) (389193326). Article processing charge was funded by the DFG and the Open Access Publishing Fund of Technische Universität Darmstadt.

\section{Author Contributions}

S.A., M.v.W. and S.B. developed the experimental idea. S.A. and M.v.W. constructed the first prototype platform, S.A. and M.v.W. performed the experiments, acquired first images of PMSs and cells and analyzed the acquired data. S.A. developed the second prototype platform, performed all experiments and the image reconstruction. S.A. and S.B. prepared the figures and wrote the manuscript. All authors interpreted the data. S.B. initiated and supervised the project.

\section{Additional Information}

Competing Interests: The authors declare no competing interests.

Publisher's note: Springer Nature remains neutral with regard to jurisdictional claims in published maps and institutional affiliations.

(c) (1) Open Access This article is licensed under a Creative Commons Attribution 4.0 International

License, which permits use, sharing, adaptation, distribution and reproduction in any medium or format, as long as you give appropriate credit to the original author(s) and the source, provide a link to the Creative Commons license, and indicate if changes were made. The images or other third party material in this article are included in the article's Creative Commons license, unless indicated otherwise in a credit line to the material. If material is not included in the article's Creative Commons license and your intended use is not permitted by statutory regulation or exceeds the permitted use, you will need to obtain permission directly from the copyright holder. To view a copy of this license, visit http://creativecommons.org/licenses/by/4.0/.

(c) The Author(s) 2019 